\title{
HANKEL TRANSFORMS AND GASP
}

\author{
BY \\ STANTON PHILIPP( $\left.{ }^{1}\right)$
}

\begin{abstract}
The inversion of the classical Hankel transform is considered from three viewpoints. The first approach is direct, and a theorem is given which allows inversion in the $(C, 1)$ sense under fairly weak hypotheses. The second approach is via Abel summability, and it is shown that inversion is possible if it is known that the Hankel transform is Abel summable and if certain critical growth conditions are satisfied. The third approach rests on the observation that $\mathrm{Abel}$ means of Hankel transforms satisfy a variant of the GASP equation in two arguments. In this setting the inversion problem becomes a boundary value problem for GASP in a quadrant of the plane with boundary values on one of the axes; a uniqueness theorem for this proble $m$ is proved which is best possible in several respects.
\end{abstract}

1. Introduction. We shall use the notations $S_{\nu}(z)=z^{1 / 2} J_{\nu}(z)$ and

$$
P_{\nu}(s, x, t)=\int_{0}^{\infty} e^{-t y} S_{\nu}(x y) S_{\nu}(s y) d y
$$

for $\nu>-1 / 2$ and $z, s, x$ and $t$ positive; here $J_{\nu}$ denotes the Bessel function of first kind and order $\nu$. If the function $f$ has domain $E \times(0, \infty)$ we use the notation $\|f(x, t)\|=\sup |f(x, t)|$, the supremum being taken over all $x$ in $E$ for fixed positive $t$; thus $\|f(x, t)\|$ is a function of $t$. The main results of this investigation are contained in the following three theorems:

Theorem 1. H. (1) $\nu>-1 / 2$ and $g$ and $h$ are locally integrable over $[0, \infty)$. (2) $b$ is finite valued and

$$
\lim _{R \rightarrow \infty} \int_{0}^{R} g(y) S_{\nu}(x y) d y=b(x)
$$

for each positive $x$.

C. $g(x)=\lim _{\boldsymbol{\lambda} \rightarrow \infty} \lambda^{-1} \int_{0}^{\lambda} d u \int_{0}^{u} b(y) S_{\nu}(x y) d y$ a.e.

Theorem 2a. H. (1) $-1 / 2<\nu<1 / 2$ and $g$ is locally integrable over $[0, \infty)$.

Received by the editors March 9, 1971 .

AMS (MOS) subject classifications (1970). Primary 44A 15, 35Q99; Secondary 35C15, $35 \mathrm{~J} 05$.

Key words and phrases. Hankel transform, GASP, locally integrable, kernel, inversion, smooth, generalized second derivative, Bessel function, Legendre function of second kind, hypergeometric function, binomial coefficient.

(1) Research sponsored in part by the Air Force Office of Scientific Research, Office of Aerospace Research, USAF, under Grant No. AFOSR-70-1942 at the University of California, Santa Cruz. The United States Government is authorized to reproduce and distribute reprints for Governmental purposes notwithstanding any copyright notation hereon. 
(2) $g(y)=o\left(y^{\nu+1 / 2}\right)$ as $y \rightarrow \infty$. (3) $b$ is a finite valued member of $L^{1}(0, \infty)$ and

$$
\lim _{t \rightarrow 0^{+}} \int_{0}^{\infty} e^{-t y} g(y) S_{,}(x y) d y=b^{(x)}
$$

for each positive $x$.

C. $g(x)=\int_{0}^{\infty} h(y) S_{\nu}(x y) d y$ a.e.

Theorem $2 \mathrm{~b}$. H. The same as Theorem $2 \mathrm{a}$ except that $\nu \geq 1 / 2$ and $g(y)=$ $o(y)$ as $y \rightarrow \infty$.

C. The same as Theorem 2a.

Theorem 3. H. $\nu>1 / 2$ and $f(x, t)$ is a $C^{2}$ solution of the equation $u_{x x}+$ $u_{t t}=\left(\nu^{2}-1 / 4\right) x^{-2} u$ in the quadrant $(0, \infty) \times(0, \infty)$. (2) $\|f(x, t)\|$ is bounded on compact subsets of $(0, \infty)$, is $o(t)$ as $t \rightarrow \infty$ and is $o\left(t^{-2}\right)$ as $t \rightarrow 0$. (3) $f(x)$ is a finite valued member of $L^{1}(0, \infty)$ and $\lim _{t \rightarrow 0} f(x, t)=f(x)$ for each positive $x$.

C. $f(x, t)=\int_{0}^{\infty} f(s) P_{\nu}(s, x, t) d s$ for all positive $x$ and $t$.

The second hypothesis of Theorem 1 expresses that $b$ is the ( $\nu$ th) Hankel transform of $g$. When $\nu= \pm 1 / 2$ the Hankel transform is a Fourier sine or cosine transform; in this case Theorem 1 is well known and is proved in [7]. Theorem 1 can be proved by adapting the argument given in [7]; since the proof is cumbersome and fairly routine, we shall omit it. If $b(x) \equiv 0$ and $-1 / 2<\nu<1 / 2$, then Theorem 1 fails if its second hypothes is is interpreted in the $(C, 1)$ sense (for an example, take $g(y)=y^{\nu+1 / 2}$ ); however, as shown in [3], this is no longer the case when $\nu=1 / 2$; what happens in this regard when $\nu>1 / 2$ seems to be unknown. A conjecture is that $(C, \nu+1 / 2)$ is appropriate in place of $(C, 1)$ and that this should fail if $(C, \nu+1 / 2+\epsilon)$ is used (I am indebted to the referee for this conjecture).

Theorem 2 is a partial generalization of Theorem 1 and is a variant of known theorems about Fourier series and transforms ([4], [10], [11]). Interestingly, Theorem 2 is best possible with respect to the growth condition on $g(y)$; that is, if $O$ is replaced by $O$ in its hypotheses, the theorem fails. To see this, take $g(y)=y^{\nu+1 / 2}$ in Theorem $2 \mathrm{a}$ and $g(y)=y S_{\nu}^{\prime}(y)$ in Theorem $2 \mathrm{~b}$; then $b \equiv 0$ but it is not the case that $g(x)=0$ a.e. We shall obtain Theorem 2 as a corollary of Theorem 3 and its proof.

We shall denote by $E_{\nu}$ the partial differential equation which occurs in Theorem 3; and we shall use the abbreviation $I=(0, \infty) \times(0, \infty)$. It is easily seen that if $f(x, t)$ satisfies $E_{\nu}$ on $l$ then $u(x, t)=x^{-\nu-1 / 2} f(x, t)$ satisfies the equation

$$
u_{x x}+u_{t t}+(2 \nu+1) x^{-1} u_{x}=0
$$

on $I$. This latter equation is the equation of generalized axially symmetric potentials (GASP). 
Theorem 3 is best possible in several respects. For example, it fails if $\nu=1 / 2$, as can be seen by taking $f(x, t)=e^{-x} \sin t$. If $f(x)$ is not finite at one point, Theorem 3 fails by virtue of the example $f(x, t)=P_{\nu}(1, x, t)$. If $f(x, t)=$ $O\left(t^{-2}\right)$ as $t \rightarrow 0$, the function $f(x, t)=P_{\nu s}(1, x, t)$ provides a counterexample. It is not known whether the hypothesis $\|f(x, t)\|=o(t)$ as $t \rightarrow \infty$ can be relaxed.

Theorem 3 was motivated by the work of V. L. Shapiro in [5] and [6]. We remark without proof that Theorem 3 is valid in the case $\nu=1 / 2$ if the first quadrant is replaced by the upper half plane and the kernel $P_{\nu}$ is replaced by the usual kernel for harmonic functions in the upper half plane.

2. Lemmas. This section is devoted to thirteen lemmas which yield Theorems 2 and 3. Of these lemmas, the first four are easy and the fifth is known. Lemmas 6-11 have more or less direct analogues in [6], but the ir correct formulation and proof offer some challenge; accordingly, we have tried to emphasize the more difficult steps in proving Lemmas 6-11, leaving the easier steps to the reader. Lemma 10 is proved in detail in arder to illustrate the role of Lemmas 8 and 9. Lemmas 12 and 13, which together imply Theorem 3, are less straightforward than the rest.

We introduce some facts and notations. The letter $A$ will be used to denote constants. Legendre's function of second kind and order $\alpha$ is given by

$$
\begin{aligned}
Q_{a}{ }^{\prime} w^{\prime}= & \pi^{1 / 2} \Gamma(\alpha+1) \Gamma(\alpha+3 / 2)^{-1}(2 w)^{-a-1} \\
& \cdot F\left(a / 2+1 / 2, \alpha / 2+1 ; \alpha+3 / 2 ; w^{-2}\right)
\end{aligned}
$$

for $a>-1$ and $w>1$; here $F$ denotes the hypergeometric series of Gauss. For positive $s, x$ and $t$ we write

$$
z=z(s, x, t)=\left(s^{2}+x^{2}+t^{2}\right) /(2 s x) .
$$

It is proved in [8] that

$$
P_{\nu}(s, x, t)=-t(\pi s x)^{-1} Q_{\nu-1 / 2}^{\prime}(z) .
$$

It is a consequence of (1) that there exist constants $A$ depending only on $\nu$ and $n$ such that

$$
\left|Q_{\nu-1 / 2}(w)\right| \leq A w^{-\nu-1 / 2}|\log (w-1)|
$$

and

$$
\left|Q_{\nu-1 / 2}^{(n)}(w)\right| \leq A w^{-\nu-1 / 2}(w-1)^{-n}
$$

hold for $\nu>-1 / 2, w>1$ and $n=1,2, \ldots$. It is clear from (1) that, when $\nu>-1 / 2$,

$$
Q_{\nu-1 / 2}(w)=O\left(w^{-\nu-1 / 2}\right) \quad \text { as } w \rightarrow \infty
$$

Lemma 1. H. (1) $f(x, t)$ is continuous for $x>0, t \geq 0$ and satisfies $E_{\nu}$ on 
I, where $\nu>-1 / 2$. (2) For each $T>0, f(x, t)$ is bounded on the strip $0 \leq t \leq T$, $x>0$. (3) $\|f(x, t)\|=o(t)$ as $t \rightarrow \infty$.

C. If $f(x, 0)=0$ for each positive $x$, then $f(x, t)=0$ on $l$.

We omit the proof of Lemma 1, which is easily carried out with the aid of the comparison function $b_{\epsilon}(x, t)=f(x, t)-\epsilon\left(x^{\nu+1 / 2} x^{-\nu+1 / 2}\right)(1+t)$, which is a solution of $E_{\nu}$ on $I$ for each $\epsilon>0$.

Lemma 2. H. (1) $\nu>-1 / 2$ and $g$ is continuous on $(0, \infty)$. (2) There exist positive numbers $\alpha$ and $\beta$ such that $g(s)=O\left(s^{\nu+3 / 2-\beta}\right)$ as $s \rightarrow \infty$ and $g(s)=$ $O\left(s^{-\nu-3 / 2+a}\right)$ as $s \rightarrow 0$. (3) $f(x, t)=\int_{0}^{\infty} g(s) P_{\nu}(s, x, t) d s$ on $I$.

C. (1) $f(x, t)$ is a $C^{2}$ solution of $E_{\nu}$ on I. (2) For each $x_{0}>0, f(x, t) \rightarrow$ $g\left(x_{0}\right)$ as $(x, t) \rightarrow\left(x_{0}, 0\right)$. (3) If $g$ is bounded on $(0, \infty)$ then $f(x, t)$ is bounded on $I$.

Since the proof of Lemma 2 is a straightforward application of (2)-(6), we omit it. An immediate consequence of Lemmas 1 and 2 is

Lemma 3. Under the bypotheses of Lemma $1, f(x, t)=\int_{0}^{\infty} f(s, 0) P_{\nu}(s, x, t) d s$ on $I$.

Lemma 4. If $\nu \geq 1 / 2$ there is a constant $A$, depending only on $\nu$, such that $\int_{0}^{\infty}\left|P_{\nu x}(s, x, t)\right| d s \leq A / t$ on $I$

Proof. Using (3) and (5) we obtain that

$$
\begin{aligned}
\int_{0}^{\infty} & \left|P_{\nu x}(s, x, t)\right| d s \\
& \leq A x^{-2} t \int_{0}^{\infty}[s z(z-1)]^{-1} d s+A t x^{-3} \int_{0}^{\infty}\left[s^{2} z(z-1)^{2}\right]^{-1}\left|s^{2}+t^{2}-x^{2}\right| d s \\
& \equiv I_{1}+I_{2} .
\end{aligned}
$$

It is easy to see that $z \geq\left(x^{2}+t^{2}\right)^{1 / 2 / x}$; and it follows that

$$
I_{1} \leq A t\left(x^{2}+t^{2}\right)^{-1 / 2} \int_{0}^{\infty}\left[(s-x)^{2}+t^{2}\right]^{-1} d s \leq A / t .
$$

A similar estimate shows that $I_{2} \leq A^{\prime} / t$ and Lemma 4 follows.

We introduce the difference quotient

$$
\begin{aligned}
& G_{2}(x, b, F) \\
& \quad=\{[x+(\nu+1 / 2) b] F(x+b)+[x-(\nu+1 / 2) b] F(x-b)-2 x F(x)\} / b^{2}
\end{aligned}
$$

and denote its $\lim$ sup and $\lim \inf$ as $b \rightarrow 0$ by $G_{2}^{*} F(x)$ and $G_{2 *} F(x)$ respectively. In general, for functions $f(x, t)$ we abbreviate

$$
f^{*}(x)=\limsup _{t \rightarrow 0^{+}} f(x, t) \text { and } f_{*}(x)=\liminf _{t \rightarrow 0^{+}} f(x, t) .
$$

Lemma 5 is proved in [4]. 
Lemma 5. H. (1) $\nu>-1 / 2,0<a<b$ and $F(x)$ is continuous on $(a, b)$. (2) On $(a, b), f(x)$ is finite valued and integrable and $G_{2 *} F(x) \leq f(x) \leq G_{2}^{*} F(x)$.

C. There are constants $p$ and $q$ such that

$$
F(x)=p x^{-2 \nu}+q+\int_{a}^{x} r^{-2 \nu-1} d r \int_{a}^{r} s^{2 \nu} f(s) d s
$$

bolds on $(a, b)$; when $\nu=0$ the term $p x^{-2 \nu}$ should be replaced by $p \log x$.

Lemma 6. H. (1) $s^{\nu+1 / 2} F(s)$ is continuous and bounded on $(0, \infty)$, where $\nu>-1 / 2$. (2) $F(x, t)=x^{-\nu-1 / 2} \int_{0}^{\infty} s^{\nu+1 / 2} F(s) P_{\nu}(s, x, t) d s$ on $I$.

C. For each positive $x, G_{2 *} F(x) \leq x \lim \sup _{t \rightarrow 0}\left[-F_{t t}(x, t)\right]$ and $G_{2}^{*} F(x) \geq$ $x \lim \inf _{t \rightarrow 0}\left[-F_{t t}(x, t)\right]$.

Proof. We observe that it suffices to prove the first inequality of the conclusion. For brevity we denote $G(t)=F\left(x_{0}, t\right), f(x, t)=-x^{\nu+1 / 2} F_{t t}(x, t)$ and $R_{\nu}(w)$ $=\left(w^{2}-1\right)^{2} Q_{\nu-1 / 2}^{\prime \prime}(w)$. Fix $\delta$ in the interval $\left(0, x_{0} / 4\right)$. It is a routine matter to establish that

$$
R_{\nu}(w)=2+\left(9 / 4-\nu^{2}\right)(w-1)+O\left((w-1)^{3 / 2}\right) \text { as } w \rightarrow 1+.
$$

In this proof we use $o(1)$ to denote any function which tends to zero with $t$. The key to this proof lies in establishing that

$$
[G(t) / t]^{\prime}+4\left(\pi t^{2}\right)^{-1} F\left(x_{0}\right) \int_{0}^{\delta / t}\left(1+s^{2}\right)^{-1} d s
$$

$$
+2 t\left(\pi x_{0}\right)^{-1} \int_{0}^{\delta} u^{2}\left(t^{2}+u^{2}\right)^{-2} G_{2}\left(x_{0}, u, F\right) d u=o(1)
$$

To prove (9) we use (1)-(8) to write

$$
\begin{aligned}
& {[G(t) / t]^{\prime}=-t x_{0}^{-\nu-s / 2} \pi^{-1} \int_{0}^{\infty} s^{\nu-3 / 2} F(s)\left(z^{2}-1\right)^{-2} R_{\nu}(z) d s} \\
& =o(1)+t x_{0}^{-\nu-5 / 2}(4 \pi)^{-1} \int_{x_{0}{ }^{-\delta}}^{x_{0}+\delta} s^{\nu-3 / 2} F(s) R_{\nu}(z)\left[(z-1)^{-1}-(z-1)^{-2}\right] d s \\
& =o(1)+t x_{0}^{-\nu-5 / 2}(4 \pi)^{-1} \\
& \left.\int_{x_{0}-\delta}^{x_{0}+\delta} s^{\nu-3 / 2} F(s)\left[\left(\nu^{2}-1 / 4\right)^{(} z-1\right)^{-1}-2(z-1)^{-2}\right] d s \\
& =o(1)+x_{0}^{-\nu-3 / 2}\left(\nu^{2}-1 / 4\right) \\
& (2 \pi)^{-1} \int_{-\delta, t}^{\delta / t}\left(x_{0}+u t\right)^{\nu-1 / 2}\left(u^{2}+1\right)^{-1} F^{\prime}\left(x_{0}+u t\right) d u \\
& \left.-2 t x_{0}^{-\nu-1 / 2} \pi^{-1} \int_{-\delta}^{\delta}\left(x_{0}+u\right)^{\nu+1 / 2}\left(u^{2}+t^{2}\right)^{-2} F^{\prime} x_{0}+u\right) d u \text {. }
\end{aligned}
$$

Now (9) is obtained from (10) by applying the binomial theorem to $\left(x_{0}+u t\right)^{\nu-1 / 2}$ and $\left(x_{0}+u\right)^{\nu+1 / 2}$. 
Now assume, to obtain a contradiction, that

$$
G_{2 *} F\left(x_{0}\right)>m>x_{0}^{-\nu+1 / 2} f^{*}\left(x_{0}\right) \text { for some real } m \text {. }
$$

It follows from (11) that there exist positive numbers $\epsilon, t_{0}$ and $\delta$ such that $0<$ $\delta<x_{0} / 4$ and

$$
G_{2}\left(x_{0}, u, F\right) \geq m+\epsilon>m-\epsilon \geq x_{0}^{-\nu+1 / 2} f\left(x_{0}, t\right)
$$

for $0<u \leq \delta$ and $0<t \leq t_{0}$. Because $G^{\prime \prime}(t)=-x_{0}^{-\nu-1 / 2} f\left(x_{0}, t\right),(12)$ yields that $G^{\prime \prime}(t) \geq(\epsilon-m) / x_{0}$ for $0<t<t_{0}$ and it follows that

(13) $G^{\prime}(t)+m t / x_{0}$ is a strictly increasing function of $t$ in the interval $\left(0, t_{0}\right)$.

By Lemma 2, $G(t)$ is continuous on $\left[0, t_{0}\right]$ if we define $G(0)=F\left(x_{0}\right)$; so, using the mean value theorem twice and (13), we conclude that for each $t$ in $\left(0, t_{0}\right)$ there exists a number $\rho$ in $(0, t)$ such that

$$
\frac{G(t)-G(0)}{t}+\frac{m t}{2 x_{0}}>\frac{G(\rho)-G(0)}{\rho}+\frac{m \rho}{2 x_{0}} \text {. }
$$

But a straightforward computation based on (9) and the first inequality in (12) shows that

$$
\limsup _{t \rightarrow 0} \frac{d}{d t}\left[\frac{G(t)-G(0)}{t}+\frac{m t}{2 x_{0}}\right]<0
$$

Now (14) and (15) are contradictory, so Lemma 6 is proved.

Lemma 7. H. (1) $f(x, t)$ is a $C^{2}$ solution of $E_{\nu}$ on $I$, with $\nu>1 / 2$. (2) $\|f(x, t)\|$ is bounded on compact subsets of $(0, \infty)$, is $o\left(t^{-2}\right)$ as $t \rightarrow 0$ and is $O\left(t^{-5}\right)$ as $t \rightarrow \infty$. (3) $F(x, t)=-x^{-\nu-1 / 2} \int_{t}^{\infty} d r \int_{r}^{\infty} f(x, s) d s$ on $I$. (4) $0<a<b$ and $\lim _{t \rightarrow 0} F(x, t)=F(x)$ exists and is continuous on $(a, b)$.

C. (1) $G_{2 *} F(x) \leq x^{-\nu+1 / 2} f^{*}(x)$ and $G_{2}^{*} F(x) \geq x^{-\nu+1 / 2} f_{*}(x)$ on $(a, b)$. (2) If $a<\alpha<\beta<b$ then $F(x, t) \rightarrow F(x)$ uniformly on $[\alpha, \beta]$ as $t \rightarrow 0$.

Proof. By Lemma 3,

$$
f(x, s)=\int_{0}^{\infty} f(u, s / 2) P_{\nu}(u, x, s / 2) d u
$$

holds for $(x, s)$ in $I$. Substitution of (16) into the formula of our third hypothesis and application of Lemma 4 allows us to differentiate with respect to $x$ under the integral sign to conclude that

$$
\begin{aligned}
& x^{\nu+1 / 2} F(x, t) \text { is a continuous solution of } E_{\nu} \text { and } \\
& x^{\nu+1 / 2} F_{t t}(x, t)=-f(x, t) \text { on } I .
\end{aligned}
$$

Next we define 


$$
b(x, t)=-\int_{t}^{\infty} d r \int_{r}^{\infty} x^{\nu+1 / 2} F(x, s) d s
$$

on I. An argument similar to that which establishes (17) yields that

$$
\begin{aligned}
& b(x, t) \text { is a continuous solution of } E_{\nu} \text { and } \\
& b_{t t}(x, t)=-x^{\nu+1 / 2} F(x, t) \text { on } I .
\end{aligned}
$$

It follows easily from our growth assumptions on $f(x, t)$ that $b(x, t)$ is bounded on $I$ and that, for $t_{1}$ and $t_{2}$ in an interval $\left(0, t_{0}\right),\left|b\left(x, t_{1}\right)-b\left(x, t_{2}\right)\right| \leq$ $A\left|t_{1}-t_{2}\right|$, where $A$ depends on $t_{0}$ but not on $x$. Therefore there is a bounded continuous function $b$ on $(0, \infty)$ such that

$$
b(x, t) \rightarrow b(x) \text { uniformly on }(0, \infty) \text { as } t \rightarrow 0 .
$$

So, by Lemma 3, we have that on $I$,

$$
b(x, t)=\int_{0}^{\infty} h(s) P_{\nu}(s, x, t) d s .
$$

Next we conclude from (21), Lemma 6 and (19) that

$$
G_{2 *}\left[x^{-\nu-1 / 2} b(x)\right] \leq x F(x) \leq G_{2}^{*}\left[x^{-\nu-1 / 2} b(x)\right] .
$$

Now (22) and Lemma 5 yield that there exist constants $p_{2}$ and $q_{2}$ such that

$$
x^{-\nu-1 / 2} b(x)=p_{2} x^{-2 \nu}+q_{2}+\int_{a}^{x} r^{-2 \nu-1} d r \int_{a}^{r} s^{2 \nu+1} F(s) d s
$$

holds for $\alpha \leq x \leq \beta$.

Let $F_{1}(x)$ be a bounded continuous function on $(0, \infty)$ which agrees with $F(x)$ on $[\alpha, \beta]$ and vanishes outside $(a, b)$. For $(x, t)$ in $I$ define

$$
\begin{aligned}
& F_{1}(x, t)=\int_{0}^{\infty} F_{1}(s)(s / x)^{\nu+1 / 2} P_{\nu}(s, x, t) d s \text { and } \\
& b_{1}(x, t)=x^{\nu+1 / 2} \int_{t}^{\infty} d r \int_{r}^{\infty} F_{1}(x, s) d s .
\end{aligned}
$$

Then arguments paralleling those of the preceding paragraph yield that (19)-(23) hold with $b_{1}$ in place of $b, F_{1}$ in place of $F$ and $p_{1}, q_{1}$ in place of $p_{2}, q_{2}$. Next we define $b_{2}(x, t)=b(x, t)-b_{1}(x, t), b_{2}(x)=b(x)-b_{1}(x), p=p_{2}-p_{1}, q=$ $q_{2}-q_{1}$. It follows that $b_{2}(x)$ is bounded and continuous on $(0, \infty)$,

$$
b_{2}(x)=p x^{-\nu+1 / 2}+q x^{\nu+1 / 2} \text { on }[\alpha, \beta]
$$

and

$$
b_{2}(x, t)=\int_{0}^{\infty} b_{2}(s) P_{\nu}(s, x, t) d s \quad \text { on } I .
$$

We propose to establish that, for $\alpha<x<\beta$,

$$
\partial^{4} b_{2}(x, t) / \partial t^{4} \rightarrow 0 \text { as } t \rightarrow 0 .
$$


Note that once (26) is proved the first conclusion of Lemma 7 follows easily.

To prove (26), fix $x_{0}$ in ( $\left.\alpha, \beta\right)$. Differentiation of (3) yields that

$$
-\pi \frac{\partial^{4}}{\partial t^{4}} P_{\nu}(s, x, t)=t^{5}(s x)^{-5} Q_{\nu-1 / 2}^{(5)}(z)+10 t^{3}(s x)^{-4} Q_{\nu-1 / 2}^{(4)}(z)
$$

$$
+15 t(x s)^{-3} Q_{\nu-1 / 2}^{(3)}(z)
$$

Choose $\delta$ so that $\alpha<x_{0}-\delta<x_{0}+\delta<\beta$. Then it follows from (27) and (5) that

$$
\begin{aligned}
& \int_{\left|s-x_{0}\right| \geq \delta} b_{2}(s) \frac{\partial^{4}}{\partial t^{4}} P_{\nu}\left(s, x_{0}, t\right) d s \quad \text { and } \\
& \int_{\left|s-x_{0}\right| \geq \delta}\left(p s^{-\nu+1 / 2}+q s^{\nu+1 / 2}\right) \frac{\partial^{4}}{\partial t^{4}} P_{\nu}\left(s, x_{0}, t\right) d s
\end{aligned}
$$

tend to zero with $t$.

By Lemma 1,

$$
e^{-t r} S_{\nu}\left(r x_{0}\right) r^{-\nu-1 / 2}=\int_{0}^{\infty} S_{\nu}(r s) r^{-\nu-1 / 2} P_{\nu}\left(s, x_{0}, t\right) d s
$$

for positive $r$ and $t$; letting $r \rightarrow 0$ we obtain by dominated convergence that

$$
\int_{0}^{\infty} s^{\nu+1 / 2} P_{\nu}\left(s, x_{0}, t\right) d s=x_{0}^{\nu+1 / 2} \text {. }
$$

We obtain by definition of $P_{\nu}$, Fubini's theorem and dominated convergence that

$$
\int_{0}^{\infty} s^{-\nu+1 / 2} P_{\nu}\left(s, x_{0}, t\right) d s=2^{1-\nu} \Gamma(\nu)^{-1} \int_{0}^{\infty} e^{-t y} y^{\nu-1} J_{\nu}\left(x_{0} y\right) d y .
$$

It follows from (30) and $[8$, p. 386] that

$$
\frac{\partial^{4}}{\partial t^{4}} \int_{0}^{\infty} s^{-\nu+1 / 2} P_{\nu}\left(s, x_{0}, t\right) d s=o(1) \quad \text { as } t \rightarrow 0 .
$$

Finally, (26) follows from (28), (29), (31) and the fact that four differentiations with respect to $t$ are permissible under the integral sign in (25).

To prove the second conclusion of Lemma 7 , it suffices to prove that if $a<\alpha_{1}<\beta_{1}<\beta$ then $F_{1}(x, t)-F(x, t) \rightarrow 0$ uniformly on $\left[\alpha_{1}, \beta_{1}\right]$ as $t \rightarrow 0$. Since $F_{1}(x, t)-F(x, t)=x^{-\nu-1 / 2} b_{2 t t}(x, t)$ on $l$, we see from (25) that we shall be done once we show that $b_{2 t t}(x, t) \rightarrow 0$ uniformly on $\left[a_{1}, \beta_{1}\right]$ as $t \rightarrow 0$; the proof of this last assertion is very similar to the proof of (26), so we omit it. This completes our proof of Lemma 7.

Smoothness is defined in [11].

Lemma 8. H. (1) The first three bypotheses of Lemma 7.

$$
G(x, t)=-\int_{t}^{\infty} d r \int_{r}^{\infty} x^{2 \nu+1} F_{x}(x, s) d s \text { on } I .
$$

C. $\lim _{t \rightarrow 0} G(x, t)=G(x)$ exists and is continuous and smooth on $(0, \infty)$. 
Proof. Fix $x_{0}>0$ and define $\theta(s)=\|f(x, s)\|+\left\|f_{x}(x, s)\right\|, \beta(x, s)=$ $x^{2 \nu+1} F_{x}(x, s)$ and

$$
\delta(s, b)=b^{-1}\left[\beta\left(x_{0}+b, s\right)+\beta\left(x_{0}-b, s\right)-2 \beta\left(x_{0}, s\right)\right] \text { for } s>0, x>0
$$

and $0<b<x_{0} / 2$. Using (16), Lemma 4 and our growth assumptions on $f(x, t)$ we obtain that

$$
\theta(s)=o\left(s^{-3}\right) \text { as } s \rightarrow 0 \text { and } \theta(s)=O\left(s^{-5}\right) \text { as } s \rightarrow \infty .
$$

Using (16) again, we see that if $K$ is a compact subset of $(0, \infty)$ then there is a constant $A$, independent of $x$ and $s$, such that

$$
|\beta(x, s)| \leq A \int_{s}^{\infty} d r \int_{r}^{\infty} \theta(u) d u
$$

for $x$ in $K$ and $s>0$. It follows easily from (32) and (33) that $G(x, t) \rightarrow G(x)$ uniformly on $K$ as $t \rightarrow 0$, where

$$
G(x)=-\int_{0}^{\infty} d r \int_{r}^{\infty} \beta(x, s) d s
$$

is continuous on $(0, \infty)$.

To prove that $G(x)$ is smooth at $x_{0}$ we write

$$
\begin{aligned}
b^{-1} \mid G & \left(x_{0}+b\right)+G\left(x_{0}-b\right)-2 G\left(x_{0}\right)\left|\leq \int_{0}^{\infty} \int_{r}^{\infty}\right| \delta(s, b) \mid d s d r \\
& =\int_{t_{0}}^{\infty} \int_{r}^{\infty}+\int_{b}^{t_{0}} \int_{t_{0}}^{\infty}+\int_{b}^{t_{0}} \int_{r}^{t_{0}}+\int_{0}^{b} \int_{r}^{b}+\int_{0}^{b} \int_{b}^{t_{0}}+\int_{0}^{b} \int_{t_{0}}^{\infty} \\
& \equiv I_{1}+\cdots+I_{6},
\end{aligned}
$$

where $0<b<t_{0}<x_{0} / 2$. By. (17) we have that

$$
\delta(s, b)=\int_{0}^{b} d y \int_{x_{0}-y}^{x_{0}+y} \frac{\partial}{\partial r}\left[r^{\nu+1 / 2} f(r, s)\right] d r .
$$

And (36) yields that

$$
|\delta(s, b)| \leq A b \theta(s)
$$

for $s>0$ and $0<b<x_{0} / 2$, where $A$ is independent of $s$ and $b$. It is now a routine matter to use (32), (33), (35) and (37) to prove that $G(x)$ is smooth at $x_{0}$; we omit the details of this and consider Lemma 8 proved. exists.

Lemma 9. H. (1) The bypotheses of Lemma 8. (2) $x_{0}>0$ and $\lim _{t \rightarrow 0} f\left(x_{0}, t\right)$

C. $\lim _{b \rightarrow 0}\left[G\left(x_{0}+b\right)-G\left(x_{0}-b\right)\right] /(2 b)=x_{0}^{2 \nu+1} F\left(x_{0}\right)$, where $F\left(x_{0}\right)$ denotes $\lim _{t \rightarrow 0} F\left(x_{0}, t\right)$.

Proof. We keep the notation of the proof of Lemma 8. Let $\Delta(s, b)=$ 
$x_{0}^{\nu+1 / 2} f\left(x_{0}, s\right)-(\Omega b)^{-1}\left[\beta\left(x_{0}+b, s\right)-\beta\left(x_{0}-b, s\right)\right]$. Then

$$
(2 b)^{-1}\left[G\left(x_{0}+b\right)-G\left(x_{0}-b\right)\right]-x_{0}^{2 \nu+1} F\left(x_{0}\right)=\lim _{t \rightarrow 0} \int_{t}^{\infty} d r \int_{r}^{\infty} \Delta(s, b) d s .
$$

It suffices to prove that

$$
\int_{0}^{\infty} d r \int_{r}^{\infty}|\Delta(s, b)| d s=o(1) \quad \text { as } b \rightarrow 0 .
$$

We write

$$
\begin{aligned}
\int_{0}^{\infty} d r & \int_{r}^{\infty}|\Delta(s, b)| d s \\
\leq & \left(\int_{t_{0}}^{\infty} \int_{r}^{\infty}+\int_{b}^{t} 0 \int_{r}^{t_{0}}+\int_{0}^{t_{0}} \int_{t_{0}}^{\infty}\right)(|\Delta(s, b)| d s d r) \\
& +\int_{0}^{b} d r \int_{r}^{t_{0}} x_{0}^{\nu+1 / 2}\left|f\left(x_{0}, s\right)\right| d s \\
& +\int_{0}^{b} d r \int_{r}^{t_{0}}(2 b)^{-1}\left|\beta\left(x_{0}+b, s\right)-\beta\left(x_{0}-b, s\right)\right| d s \\
\equiv & J_{1}+\cdots+J_{5} .
\end{aligned}
$$

By the mean value theorem there exist numbers $x$ and $r$ in the interval $\left(x_{0}-b\right.$, $\left.x_{0}+b\right)$ such that $\Delta(s, b)=x_{0}^{\nu+1 / 2} f\left(x_{0}, s\right)-\beta_{x}(x, s)=x_{0}^{\nu+1 / 2} f\left(x_{0}, s\right)-$ $x^{\nu+1 / 2} f(x, s)=\left(x_{0}-x\right) \partial\left[r^{\nu+1 / 2} f(r, s)\right] / \partial r$; and it follows that

$$
|\Delta(s, b)| \leq A b \theta(s) \text {. }
$$

Again by the mean value theorem there exists a number $y$ in $\left(x_{0}-b, x_{0}+b\right)$ such that

$$
\begin{aligned}
(2 b)^{-1} \mid \beta\left(x_{0}+b, s\right)- & \beta\left(x_{0}-b, s\right)|=| \beta_{x}(y, s) \mid \\
= & \left|y^{\nu+1 / 2} f(y, s)\right| \leq A\|f(y, s)\| \cdot
\end{aligned}
$$

Then, as in the proof of Lemma 8 , it is a routine matter to establish (38), using (32), (33), (40) and (41); we again omit the details and consider Lemma 9 proved.

Lemma 10. H. (1) The first three bypotheses of Lemma 7. (2) $f(s)$ is locally integrable on $[0, \infty)$ and $\lim _{t \rightarrow 0} f(x, t)=f(x)$ exists for each positive $x$.

(3) $\lim _{t \rightarrow 0} F(x, t)=F(x)$ is continuous on $(a, b)$ where $0<a<b$.

C. $F(x)$ is continuous on $[a, b]$.

Proof. By Lemma 5 and the first conclusion of Lemma 7, there exist constants $p$ and $q$ such that

$$
F(x)=p x^{-2 \nu}+q+\int_{a}^{x} r^{-2 \nu-1} d r \int_{a}^{r} s^{\nu+1 / 2} f(s) d s \quad \text { on }(a, b) .
$$


Therefore $F(a+)=\lim _{x \rightarrow a+} F(x)$ exists. It is a consequence of (19) that $G_{x}(x, t)$ $=x^{2 \nu+1} F(x, t)$ on $I$; therefore $G(\beta, t)-G(\alpha, t)=\int_{a}^{\beta} x^{2 \nu+1} F(x, t) d x$ holds for $a<\alpha<\beta<b$ and $t>0$. Letting $t$ tend to zero we obtain that $G(\beta)-G(\alpha)=$ $\int_{a}^{\beta} x^{2 \nu+1} F(x) d x$ by Lemma 8 and the second conclusion of Lemma 7 . Since $G(x)$ is continuous at $a$ (by Lemma 8 ) and $F(a+)$ exists, we may let $a \rightarrow a+$ to obtain that $G(\beta)-G(a)=\int_{a}^{\beta} x^{2 \nu+1} F(x) d x$ for $a \leq \beta<b$. Therefore $[G(\beta)-G(a)] /(\beta-a)$ $\rightarrow a^{2 \nu+1} F(a+)$ as $\beta \rightarrow a+$; since $G(x)$ is smooth at $a$ (Lemma 8 ), it follows that $G^{\prime}(a)=a^{2 \nu+1} F(a+)$. By the preceding sentence and Lemma 9 we see that $F(a+)=F(a)$. The proof that $F(b-)=F(b)$ is similar. So Lemma 10 is established.

Lemma 11. Under the first two bypotheses of Lemma $10, \lim _{t \rightarrow 0} F(x, t)=$ $F(x)$ is continuous on $(0, \infty)$.

Proof. Let $0<a<b$ and let $Z$ be the set of points in $(a, b)$ at which $F(x)$ is not continuous. Assume, to get a contradiction, that $Z$ is nonempty. We choose a sequence $t_{1}>t_{2}>\cdots$ such that $t_{n} \rightarrow 0$ and $\left|f(x, t)-f\left(x, t_{n}\right)\right| \leq 1$ whenever $x \in[a, b]$ and $t \in\left[t_{n}+1, t_{n}\right]$. By a familiar consequence of Baire's category theorem there exist an open subinterval $J$ of $(a, b)$ and a number $M$ such that $J \bar{Z}$ is nonempty and $\left|f\left(x, t_{n}\right)\right| \leq M$ holds for $x$ in $J \bar{Z}$ and $n=1,2, \cdots$. It follows that if $x$ is in $J \bar{Z}$ then $\left|F\left(x, t_{n}\right)-F\left(x, t_{m}\right)\right| \leq A\left|t_{n}-t_{m}\right|$. Therefore $F\left(x, t_{n}\right)$ converges uniformly on $J \bar{Z}$ as $n \rightarrow \infty$; so $F(x)$ is continuous on the subspace $J \bar{Z}$ of $(0, \infty)$.

Next fix $a>0$ and define $F_{1}(x)=\int_{a}^{x} r^{-2 \nu-1} d r \int_{a}^{r} s^{\nu+1 / 2} f(s) d s$ and $F_{2}(x)=$ $F(x)-F_{1}(x)$ on $(0, \infty)$. If $K$ is any subinterval of $J$ which is contiguous to $\bar{Z}$ then it follows from Lemmas 5,7 and 10 that there exist constants $p$ and $q$ (depending on $K$ ) such that $F_{2}(x)=p x^{-2 \nu}+q$ for $x$ in $\bar{K}$. And it follows from the preceding sentence and the previously noted fact that $F(x)$ is continuous on the space $J \bar{Z}$ that $F(x)$ is continuous on $J$, a contradiction. This proves Lemma 11.

Lemma 12. H. (1) The bypotheses of Theorem 3. (2) There exist $C^{2}$ solutions $f_{1}(x, t)$ and $f_{2}(x, t)$ of $E_{\nu}$ such that

(i) $f(x, t)=f_{1}(x, t)+f_{2}(x, t)$ on $l$;

(ii) $f_{2}(x, t)$ is bounded on $I$ and $\lim _{(x, t) \rightarrow\left(x_{0}, 0\right)} f_{2}(x, t)=f_{2}\left(x_{0}\right)$ exists for each $x_{0}>0$;

(iii) $\left\|f_{1}(x, t)\right\|=O\left(t^{-5}\right)$ as $t \rightarrow \infty$.

C. The conclusion of Theorem 3 .

Proof. Define $g(x, t)=-\int_{t}^{\infty} d r \int_{r}^{\infty} f_{1}(x, s) d s$ and $b(x, t)=-\int_{t}^{\infty} d r \int_{r}^{\infty} g(x, s) d s$ on $I$. Let $g(x), b(x)$ and $f_{1}(x)$ denote the limits as $t \rightarrow 0$ of $g(x, t), b(x, t)$ and $f_{1}(x, t)$. Let $a$ be fixed and positive. Then by Lemmas 11,7 , and 5 there exist constants $p$ and $q$ such that

$$
g(x)=p x^{\nu+1 / 2}+q x^{-\nu+1 / 2}+x^{\nu+1 / 2} \int_{a}^{x} r^{-2 \nu-1} d r \int_{0}^{r} s^{\nu+1 / 2} f_{1}(s) d s
$$


holds for $x>0$. By the proof of Lemma 7 we have that $h(x)$ is bounded and continuous on $(0, \infty)$ and that

$$
b^{\prime \prime}(s)-\left(\nu^{2}-1 / 4\right) s^{-2} b(s)=g(s) \text { for } s>0
$$

and

$$
g(x, t)=-b_{t t}(x, t)=-\int_{0}^{\infty} b(s) P_{\nu t t}(s, x, t) d s \text { on } I .
$$

Using (43), (44), the fact that $P_{\nu}$ satisfies $E_{\nu}$ on $I$, and integration by parts we obtain that

$$
\begin{aligned}
g(x, t)= & \int_{0}^{\infty} g(s) P_{\nu}(s, x, t) d s \\
& +\int_{0}^{\infty}\left[b(s) P_{\nu s s}(s, x, t)-b^{\prime \prime}(s) P_{\nu}(s, x, t)\right] d s \\
= & \int_{0}^{\infty} g(s) P_{\nu}(s, x, t) d s .
\end{aligned}
$$

Now define

$$
F_{2}(s)=s^{\nu+1 / 2} \int_{a}^{s} r^{-2 \nu-1} d r \int_{0}^{r} u^{\nu+1 / 2} f_{2}(u) d u
$$

and

$$
F(s)=s^{\nu+1 / 2} \int_{\alpha}^{s} r^{-2 \nu-1} d r \int_{0}^{r} u^{\nu+1 / 2} f(u) d u
$$

for $s>0$. Since $f_{1}(x, t)=-g_{t t}(x, t)$, it follows from (42) and (45) that

$$
f_{1}(x, t)=-q \frac{\partial^{2}}{\partial t^{2}} \int_{0}^{\infty} s^{-\nu+1 / 2} P_{\nu}(s, x, t) d s
$$

$$
+\frac{\partial^{2}}{\partial t^{2}} \int_{0}^{\infty}\left[F_{2}(s)-F(s)\right] P_{\nu}(s, x, t) d s .
$$

From Lemma 3 and partial integration we obtain that

$$
\begin{aligned}
f_{2}(x, t) & =\int_{0}^{\infty} f_{2}(s) P_{\nu}(s, x, t) d s=-\int_{0}^{\infty} F_{2}(s) P_{\nu t t}(s, x, t) d s \\
& =\frac{-\partial^{2}}{\partial t^{2}} \int_{0}^{\infty} F_{2}(s) P_{\nu}(s, x, t) d s .
\end{aligned}
$$

Adding (46) and (47) and then integrating by parts, we have that

$$
f(x, t)=-q \frac{\partial^{2}}{\partial t^{2}} \int_{0}^{\infty} s^{-\nu+1 / 2} P_{\nu}(s, x, t) d s+\int_{0}^{\infty} f(s) P_{\nu}(s, x, t) d s .
$$

Finally, we conclude from (48), (30) [8, p. 386] and the assumption that $\|f(x, t)\|=o\left(t^{-2}\right)$ as $t \rightarrow 0$ that $q=0$. And Lemma 12 is immediate from (48) and the fact that $q=0$. 
Lemma 13. The bypotheses of Theorem 3 imply the second bypothes is of Lemma 12.

Proof. For $(x, t)$ in $l$ we write $g_{0}(x, t)=f(x, t)$ and define

$$
g_{n}(x, t)=e^{t} \int_{t}^{\infty} e^{-s} g_{n-1}(x, s) d s, \quad n=1,2, \ldots
$$

By Lemma 3, $f(x, t)=\int_{0}^{\infty} f\left(s, t_{0}\right) P_{\nu}\left(s, x, t-t_{0}\right) d s$ holds for $t>t_{0}>0$ and $x>0$; it follows that $\|f(x, t)\|=O(1)$ as $t \rightarrow \infty$. The following facts are easy consequences of this last remark and the hypotheses of Theorem 3:

$$
\begin{aligned}
& g_{n}(x, t) \text { is a } C^{2} \text { solution of } E_{\nu} \text { on } I \text { for } n \geq 0 ; \\
& \left\|g_{n}(x, t)\right\|=O(1) \text { as } t \rightarrow \infty \text { for } n \geq 0 ; \\
& \left\|g_{1}(x, t)\right\|=o\left(t^{-1}\right) \text { as } t \rightarrow 0,\left\|g_{2}(x, t)\right\|=o(\log 1 / t) \text { as } t \rightarrow 0, \text { and } g_{n}(x, t)
\end{aligned}
$$

is bounded on $I$ for $n \geq 3$;

for $n \geq 3, g_{n}(x, t)$ converges uniformly on compact subsets of $(0, \infty)$ as $t \rightarrow 0$; $g_{n t}(x, t)=g_{n}(x, t)-g_{n-1}(x, t)$ for $n \geq 1$ and $(x, t)$ in $I$.

It follows that

$$
f(x, t)=\sum_{j=0}^{n}(-1)^{j}\left(\begin{array}{l}
n \\
j
\end{array}\right) \frac{\partial^{j}}{\partial t^{j}} g_{n}(x, t)
$$

and

$$
\frac{\partial^{j}}{\partial t^{j}} g_{n}(x, t)=\sum_{k=0}^{j}(-1)^{k}\left(\begin{array}{l}
j \\
k
\end{array}\right) g_{n-k}(x, t)
$$

for $(x, t)$ in $I$ and $0 \leq j \leq n$. We as sume henceforth that $n \geq 7$ and define

$$
f_{2}(x, t)=\sum_{j=0}^{n-3}(-1)^{j}\left(\begin{array}{l}
n \\
j
\end{array}\right) \frac{\partial^{j}}{\partial t^{j}} g_{n}(x, t)
$$

and $f_{1}(x, t)=f(x, t)-f_{2}(x, t)$. It is clear from the above that $f_{2}(x, t)$ is as required. So we shall be done once we show that

$$
\left\|\frac{\partial^{j}}{\partial t^{j}} g_{n}(x, t)\right\|=O\left(t^{-j}\right) \text { as } t \rightarrow \infty
$$

for $j=0,1,2, \cdots$.

For $j=0,(51)$ is true by Lemma 3. Since $P_{\nu t}(s, x, t)=t^{-1} P_{\nu}(s, x, t)-$ $\pi^{-1} t^{2}(s x)^{-2} Q_{\nu-1 / 2}^{\prime \prime}(z),(51)$ will be true by induction on $j$ if we can prove that

$$
\left\|\int_{0}^{\infty}\left|t^{m}(s x)^{-m} Q_{\nu-1 / 2}^{(m)}(z)\right| d s\right\|=O\left(t^{1-m}\right) \text { as } t \rightarrow \infty
$$

for $m=2,3,4, \cdots$. But (52) follows easily from (5). This completes our proof of Lemma 13. 
3. Proof of Theorem 2. Since Theorem $2 \mathrm{~b}$ is known when $\nu=1 / 2$ [10] and follows from Theorem 3 otherwise, we need to prove Theorem $2 \mathrm{a}$.

Let $g_{1}(y)=0$ for $0<y<1$ and $g_{1}(y)=g(y)$ for $y \geq 1$. For $(x, t)$ in $I$ define

$$
F(x, t)=x^{-\nu-1 / 2} \int_{0}^{\infty} e^{-t y} y^{-2} g_{1}(y) S_{\nu}(x y) d y \quad \text { and } F(x)=\lim _{t \rightarrow \infty} F(x, t) .
$$

By Theorem 1,

$$
g_{1}(y)=-y^{2} \lim _{\lambda \rightarrow \infty} \lambda^{-1} \int_{0}^{\lambda} d u \int_{0}^{u} F(s) s^{\nu+1 / 2} S_{\nu}(s y) d s \text { a.e. }
$$

Integrating twice by parts in (53) and using Lemmas 5 and 6 we obtain that there exists a constant $p$ such that

$$
g(y)=p y^{\nu+1 / 2}+\int_{0}^{\infty} h(x) S_{\nu}(x y) d y \text { a.e.; }
$$

it follows from our assumptions on $g$ and $b$ that $p=0$, which proves Theorem 2a.

I wish to express my sincere gratitude to Professor V. L. Shapiro for his helpful comments on this work.

\section{BIBLIOGRA PHY}

1. S. Bochner, Vorlesungen über Fouriersche Integrale, Akademische Verlagsgesellschaft, Leipzig, 1932; English transl., Ann. of Math. Studies, no. 42, Princeton Univ. Press, Princeton, N. J., 1959. MR 21 \#5851.

2. G. H. Hardy, Divergent series, Clarendon Press, Oxford, 1949. MR 11, 25.

3. A. C. Offord, On the uniqueness of the representation of a function by a trigonometric integral, Proc. London Math. Soc. (2) 42 (1937), 422-480.

4. P. M. Owen, The Riemannian theory of Hankel transforms, Proc. London Math. Soc. (2) 39 (1935), 295-320.

5. V. L. Shapiro, The uniqueness of functions harmonic in the interior of the unit disk, Proc. London Math. Soc. (3) 13 (1963), 639-652. MR 27 \#5916.

6. - The uniqueness of solutions of the heat equation in an infinite strip, Trans. Amer. Math. Soc. 125 (1966), 326-361. MR 34 \#1727.

7. E. C. Titchmarsh, Introduction to the theory of Fourier integrals, Clarendon Press, Oxford, 1937.

8. G. N. Watson, A treatise on the theory of Bessel functions, Cambridge Univ. Press, Cambridge; Macmillan, New York, 1922.

9. E. T. Whittaker and G. N. Watson, A course of modern analysis, Cambridge Univ. Press, New York, 1927.

10. A. Zygmund, On trigonometric integrals, Ann. of Math. (2) 48 (1947), 393-440. MR 9, 88.

11. - - Trigonometrical series. Vol. 1, 2nd rev. ed., Cambridge Univ. Press, New York, 1959. MR $21 \# 6498$.

DEPARTMENT OF MATHEMATICS, UNIVERSITY OF CALIFORNIA, SANTA CRUZ, CALIFORNIA 95060 